\title{
Blocking light in compact Riemannian manifolds
}

\author{
JEAN-FRANÇOIS LAFONT \\ BENJAMIN SCHMIDT
}

\begin{abstract}
We study compact Riemannian manifolds $(M, g)$ for which the light from any given point $x \in M$ can be shaded away from any other point $y \in M$ by finitely many point shades in $M$. Compact flat Riemannian manifolds are known to have this finite blocking property. We conjecture that amongst compact Riemannian manifolds this finite blocking property characterizes the flat metrics. Using entropy considerations, we verify this conjecture amongst metrics with nonpositive sectional curvatures. Using the same approach, K Burns and E Gutkin have independently obtained this result. Additionally, we show that compact quotients of Euclidean buildings have the finite blocking property.

On the positive curvature side, we conjecture that compact Riemannian manifolds with the same blocking properties as compact rank one symmetric spaces are necessarily isometric to a compact rank one symmetric space. We include some results providing evidence for this conjecture.
\end{abstract}

$53 \mathrm{C} 22 ; 53 \mathrm{C} 20,53 \mathrm{~B} 20$

\section{Introduction}

To what extent does the collision of light determine the global geometry of space? In this paper we study compact Riemannian manifolds with this question in mind. Throughout, we assume that $(M, g)$ is a smooth, connected, and compact manifold without boundary equipped with a smooth Riemannian metric $g$. Unless stated otherwise, a geodesic segment $\gamma$ refers to a unit speed paramaterization of a geodesic in $M$. Notationally, we will write such a paramaterized segment as $\gamma:\left[0, L_{\gamma}\right] \rightarrow M$, where $L_{\gamma}$ is the length of the segment $\gamma$. By the interior of a geodesic segment $\gamma$ we mean the set $\operatorname{int}(\gamma):=\gamma\left(\left(0, L_{\gamma}\right)\right) \subset M$.

Definition (Light) Let $X, Y \subset(M, g)$ be two nonempty subsets, and let $G_{g}(X, Y)$ denote the set of geodesic segments $\gamma \subset M$ with initial point $\gamma(0) \in X$ and terminal point $\gamma\left(L_{\gamma}\right) \in Y$. The light from $X$ to $Y$ is the set

$$
L_{g}(X, Y)=\left\{\gamma \in G_{g}(X, Y) \mid \operatorname{int}(\gamma) \cap(X \cup Y)=\varnothing\right\} .
$$


Definition (Blocking Set) Let $X, Y \subset M$ be two nonempty subsets. A subset $B$ is a blocking set for $L_{g}(X, Y)$ provided that for every $\gamma \in L_{g}(X, Y)$,

$$
\operatorname{int}(\gamma) \cap B \neq \varnothing .
$$

In this paper we focus on compact Riemannian manifolds for which the light between pairs of points in $M$ is blocked by a finite set of points. We remark that by a celebrated theorem of Serre [24], $G_{g}(x, y)$ is infinite when $x, y \in M$ are two distinct points. In contrast, $L_{g}(x, y) \subset G_{g}(x, y)$ may or may not be an infinite subset. For example, in the case of a round metric on a sphere, $|L(x, y)|$ is infinite only when $x=y$ or $x$ and $y$ are an antipodal pair.

Definition (Blocking Number) Let $x, y \in M$ be two (not necessarily distinct) points in $(M, g)$. The blocking number $b_{g}(x, y)$ for $L_{g}(x, y)$ is defined by

$$
b_{g}(x, y)=\inf \left\{n \in \mathbb{N} \cup\{\infty\} \mid L_{g}(x, y) \text { is blocked by } n \text { points }\right\} .
$$

Definition (Finite Blocking Property) A compact Riemannian manifold $(M, g)$ is said to have finite blocking if $b_{g}(x, y)<\infty$ for every $(x, y) \in M \times M$. When $(M, g)$ has finite blocking and the blocking numbers are uniformly bounded above, $(M, g)$ is said to have uniform finite blocking.

The finite blocking property seems to have originated in the study of polygonal billiard systems and translational surfaces (see Fomin [7], Gutkin [9; 10; 11], Heimer and Snurnikov [13], and Monteil [16; 17; 18; 19]). Our motivation comes from the following theorem (see [7], [9, Lemma 1], or Gutkin and Schroeder [12, Proposition 2]):

Theorem Compact flat Riemannian manifolds have uniform finite blocking.

We believe the following is true:

Conjecture Let $(M, g)$ be a compact Riemannian manifold with finite blocking. Then $g$ is a flat metric.

There is a natural analogue of (uniform) finite blocking for general geodesic metric spaces. We provide an extension of the above theorem in Section 5:

Theorem 1 Compact quotients of Euclidean buildings have uniform finite blocking.

As evidence for the above conjecture we prove the following theorem in Section 4: 
Theorem 2 Let $(M, g)$ be a compact nonpositively curved Riemannian manifold with the finite blocking property. Then $g$ is a flat metric.

This theorem is a consequence of a well known result about nonpositively curved manifolds and the next theorem relating the finite blocking property to the topological entropy of the geodesic flow:

Theorem 3 Let $(M, g)$ be a compact Riemannian manifold without conjugate points. If $h_{\text {top }}(g)>0$, then $b_{g}(x, y)=\infty$ for every $(x, y) \in M$. In other words, given any pair of points $x, y \in M$ and a finite set $F \subset M-\{x, y\}$, there exists a geodesic segment connecting $x$ to $y$ and avoiding $F$.

Working independently and using a similar approach K Burns and E Gutkin have also obtained Theorem 3 as well as the following [4]:

Theorem (Burns and Gutkin) Let $(M, g)$ be a compact Riemannian manifold with uniform finite blocking. Then $h_{\text {top }}(g)=0$ and the fundamental group of $M$ is virtually nilpotent.

In Section 2, we define regular finite blocking by imposing a continuity and separation hypothesis on blocking sets. We show that manifolds with regular finite blocking have uniform finite blocking and are conjugate point free. Combining this result with the previous theorem of K Burns and E Gutkin, and recent work of N D Lebedeva [14] yields:

Theorem 4 Let $(M, g)$ be a compact Riemannian manifold with regular finite blocking. Then $g$ is a flat metric.

Blocking light is also interesting in the context of the nonnegatively curved compact type locally symmetric spaces. In [12], Gutkin and Schroeder show the following:

Theorem (Gutkin and Schroeder) Let $(M, g)$ be a compact locally symmetric space of compact type with $\mathbb{R}$-rank $k \geq 1$. Then $b_{g}(x, y) \leq 2^{k}$ for almost all $(x, y) \in M \times M$.

We refer the reader to [12] for a more precise formulation and discussion of this result. On the positively curved side, we focus on the blocking properties of the compact rank one symmetric spaces or CROSSes. The CROSSes are classified and consist of the round spheres $\left(S^{n}, \mathrm{can}\right)$, the projective spaces $\left(K \mathbb{P}^{n}\right.$, can $)$ where $K$ denotes one of $\mathbb{R}, \mathbb{C}$, or $\mathbb{H}$, and the Cayley projective plane $\left(C a \mathbb{P}^{2}\right.$, can $)$ where "can" denotes a symmetric metric. The CROSSes all satisfy the following blocking property: 
Definition (Cross Blocking) A compact Riemannian manifold $(M, g)$ is said to have cross blocking if

$$
0<d(x, y)<\operatorname{Diam}(M, g) \Longrightarrow b_{g}(x, y) \leq 2 .
$$

Just as with finite blocking, we also define regular cross blocking by imposing a continuity and separation hypothesis on blocking sets. In addition to cross blocking, round spheres also satisfy the following blocking property:

Definition (Sphere Blocking) A compact Riemannian manifold $(M, g)$ is said to have sphere blocking if $b_{g}(x, x)=1$ for every $x \in M$.

This is a blocking interpretation of "antipodal points"; we think of the single blocker for $L_{g}(x, x)$ as being antipodal to $x$. We believe the following is true:

Conjecture A compact simply connected Riemannian manifold $(M, g)$ has cross blocking if and only if $(M, g)$ is isometric to a simply connected compact rank one symmetric space. In particular, $(M, g)$ has cross blocking and sphere blocking if and only if $(M, g)$ is isometric to a round sphere.

As support for this conjecture, we prove the following theorems in Section 3:

Theorem 5 Let $\left(S^{2}, g\right)$ be a metric on the two sphere with cross blocking and sphere blocking. Then a shortest periodic geodesic is simple with period $2 \operatorname{Diam}\left(S^{2}, g\right)$.

Theorem 6 Let $\left(M^{2 n}, g\right)$ be an even dimensional manifold with positive sectional curvatures and regular cross blocking. Then $(M, g)$ is a Blaschke manifold. If in addition $M$ is diffeomorphic to a sphere or has sphere blocking, then $(M, g)$ is isometric to a round sphere.

Theorem 7 Let $(M, g)$ be a compact Riemannian manifold with regular cross blocking, sphere blocking, and which does not admit a nonvanishing line field. Then $(M, g)$ is isometric to an even dimensional round sphere.

Acknowledgements We owe our gratitude to Keith Burns; Keith pointed out a subtlety regarding Theorem 3 (Proposition 4.2 below) that we originally missed during the earlier stages of this work. We thank Ralf Spatzier for numerous helpful discussions. We would also like to thank Eugene Gutkin, Pilar Herreros and Tracy Payne for pointing out some inaccuracies in an early draft of this paper. 
The first author would like to thank the École Normale Supérieure (Lyon) for their hospitality. The work of the first author was partially supported by the National Science Foundation under grant DMS-0606002. The second author would like to thank The Ohio State University mathematics department for their hospitality. This research was partially conducted during the period the second author was employed by the Clay Mathematics Institute as a Liftoff Fellow.

\section{Finite blocking and conjugate points}

For the reader's convenience, we begin with the definition of a conjugate point in a compact Riemannian manifold $(M, g)$. We let $T M$ (resp. $U M$ ) denote the tangent bundle (resp. unit tangent bundle) of $M$ and denote the fibers above a point $p \in M$, by $T_{p} M$ and $U_{p} M$. For a point $p \in M$, the exponential map

$$
\exp _{p}: T_{p} M \rightarrow M
$$

is everywhere defined by completeness.

Definition (Conjugate Point) A point $q=\exp _{p}(v) \in M$ is conjugate to $p$ along the unit speed geodesic $\gamma_{v}:[0,\|v\|] \rightarrow M$ with initial condition $\frac{v}{\|v\|} \in U_{p}(M) \subset U M$ if $d\left(\exp _{p}\right)_{v}$ is not of full rank.

In [25], F Warner describes the conjugate locus of singular points $C(p) \subset T_{p}(M)$ for the exponential map $\exp _{p}$. A point $v \in C(p)$ is said to be regular if there exists a neighborhood $U$ of $v$ such that each ray emanating from the origin in $T_{p}(M)$ intersects at most one point in $C(p) \cap U$. The order of a point $v \in C(p)$ is defined to be the dimension of the kernel of $d\left(\exp _{p}\right)_{v}$. Warner shows that the set of regular points $C^{R}(p) \subset C(p)$ is an open dense subset of $C(p)$ which (if nonempty) forms a codimension one submanifold of $T_{p}(M)$. Moreover, the order of points is constant in each connected component of $C^{R}(p)$ and there are normal forms (depending on the order of the point) for the exponential map in a neighborhood of each regular point. From these normal forms, it follows that the preimage under the exponential map of a regular conjugate point of order more than one is indiscrete. It appears that regular points of order more than one are rare in Riemannian manifolds (see Warner [26]). The next proposition shows that there are no such conjugate points in Riemannian manifolds with the finite blocking property.

Proposition 2.1 Suppose that $(M, g)$ is a compact Riemannian manifold with finite blocking. Then for each $p \in M$, point preimages of $\exp _{p}$ are discrete subsets of $T_{p} M$. 
Proof Suppose not. Then there are points $p, q \in M$ and a sequence of vectors $\left\{v_{i}\right\} \subset$ $\exp _{p}^{-1}(q)$ converging to a vector $v_{\infty} \in \exp _{p}^{-1}(q) \subset T_{p}(M)$. Let $B=\left\{b_{1}, \ldots, b_{k}\right\} \subset M$ be a finite blocking set for $L_{g}(p, q)$. Note that any geodesic segment $\gamma \in G_{g}(p, q)$ contains a subsegment $\gamma^{\prime} \in L_{g}(p, q)$. It follows that for each index $i$, there is a well defined time $t_{i} \in(0,1)$, given by

$$
t_{i}=\inf \left\{t \in(0,1) \mid \exp _{p}\left(t v_{i}\right) \in B\right\} .
$$

After possibly relabeling blockers and passing to a subsequence, we may assume that $\exp _{p}\left(t_{i} v_{i}\right)=b_{1}$ for all $i \in \mathbb{N}$. A subsequence of the vectors $\left\{t_{i} v_{i}\right\}$ converge to a vector $t_{\infty} v_{\infty}$ and by continuity of the exponential map, $\exp _{p}\left(t_{\infty} v_{\infty}\right)=b_{1}$. This shows that the point $b_{1}$ is a sooner conjugate point to $p$ along the geodesic ray $\gamma(t)=\exp _{p}\left(t v_{\infty}\right)$ than is the point $q$. By repeating this argument, there is always a sooner conjugate point, contradicting the fact that conjugate points are discrete along a geodesic.

We expect that compact Riemannian manifolds with the finite blocking property will never have conjugate points. Next we impose some restrictions on blocking sets and show that the light between conjugate points cannot be finitely blocked by such sets. For a compact Riemannian manifold $(M, g)$, let $M^{\prime} \subset M \times M$ be the subset of points for which $b_{g}<\infty, T^{\prime} \subset T M$ be the subset of vectors $(p, v) \in T M$ for which $\left(p, \exp _{p}(v)\right) \in M^{\prime}$, and let $\mathcal{F}(M)$ denote the set of finite subsets of $M$. A blocking function for $(M, g)$ is a symmetric map $\mathcal{B}: M^{\prime} \rightarrow \mathcal{F}(M)$ such that for each $(x, y) \in M^{\prime}, \mathcal{B}(x, y)$ is a finite blocking set for $L_{g}(x, y)$. Given a blocking function $\mathcal{B}$ we define the first blocking time $t_{\mathcal{B}}: T^{\prime} \rightarrow(0,1)$ by

$$
t_{\mathcal{B}}(p, v)=\inf \left\{t \in(0,1) \mid \exp _{p}(t v) \in \mathcal{B}\left(p, \exp _{p}(v)\right)\right\}
$$

Definition (Continuous Blocking) We say that a closed Riemannian manifold $(M, g)$ has continuous blocking if there is a blocking function $\mathcal{B}$ for which the first blocking time $t_{\mathcal{B}}: T^{\prime} \rightarrow(0,1)$ is continuous.

Definition (Separated Blocking) We say that a blocking function $\mathcal{B}$ is separated if there exists an $\epsilon>0$ such that the $\epsilon$-neighborhoods of blocking points in each finite blocking set $\mathcal{B}(x, y) \subset M$ are disjoint.

Lemma 2.2 Let $\mathcal{B}$ be a separated blocking function for a compact Riemannian manifold $(M, g)$. Then the cardinalities of blocking sets defined by $\mathcal{B}$ are uniformly bounded above. In particular, compact Riemannian manifolds with finite blocking and a separated blocking function have uniform finite blocking. 
Proof Suppose that the blocking sets defined by $\mathcal{B}$ are $\epsilon$-separated. An upper bound $K_{\max }$ for the sectional curvatures yields a lower bound $C:=C\left(K_{\max }, \epsilon\right)>0$ for the volume of balls of radius $\epsilon$ in $M$. Therefore, there are at $\operatorname{most} \operatorname{vol}(M, g) / C$ disjoint balls of radius $\epsilon$ in $M$, concluding the proof.

Proposition 2.3 Let $(M, g)$ be a compact Riemannian manifold with a blocking function $\mathcal{B}$ that is both continuous and separated. Let $p \in M$ and suppose that $U$ is an open subset of $T_{p}(M)$ consisting of vectors from $T^{\prime} \cap T_{p}(M)$. Then no point in $\exp _{p}(U) \subset M$ is a first conjugate point to the point $p$.

Proof Suppose not. Then there is a vector $v \in U$ for which $\exp _{p}(v)$ is the first conjugate point to $p$ along the geodesic $\gamma(t):=\exp _{p}(t v), t \in[0,1]$. It is well known (see eg, Warner [25]) that $\exp _{p}$ is not one to one in any neighborhood of $v$. Let $B_{i}$ be a sequence of balls centered at $v$ and contained in $U$ with radii decreasing to zero. For each $i$, choose distinct points $x_{i}, y_{i} \in B_{i}$ with $\exp _{p}\left(x_{i}\right)=\exp _{p}\left(y_{i}\right):=q_{i}$. Let $\mathcal{B}$ be a continuous and separated blocking function, and let $l_{i}:=\exp _{p}\left(t_{\mathcal{B}}\left(x_{i}\right) x_{i}\right)$ and $r_{i}:=\exp _{p}\left(t_{\mathcal{B}}\left(y_{i}\right) y_{i}\right)$ be the associated first blocking points in $\mathcal{B}\left(p, q_{i}\right)$. By continuity of $\mathcal{B}$, the sequences $\left\{l_{i}\right\}$ and $\left\{r_{i}\right\}$ both converge to $\exp _{p}\left(t_{\mathcal{B}}(v) v\right)$. By the separatedness of $\mathcal{B}$, it follows that $l_{i}=r_{i}$ for all sufficiently large indices, whence $\exp _{p}\left(t_{\mathcal{B}}(v) v\right)$ is a sooner conjugate point along $\gamma$, a contradiction.

When a compact Riemannian manifold $(M, g)$ has finite (resp. cross) blocking and a continuous and separated blocking function, we shall say that $(M, g)$ has regular finite (resp. regular cross) blocking.

Corollary 2.4 Let $\left(M^{n}, g\right)$ be a compact Riemannian manifold with regular finite blocking. Then $(M, g)$ has uniform finite blocking and is conjugate point free. In particular, the universal cover of $M$ is diffeomorphic to $\mathbb{R}^{n}$

Proof Since $(M, g)$ has finite blocking, $T^{\prime}=T M$. By Lemma 2.2, $(M, g)$ has uniform finite blocking and by Proposition 2.3, $(M, g)$ is conjugate point free. The second statement is Hadamard's theorem.

Corollary 2.5 Let $(M, g)$ be a compact Riemannian manifold with regular cross blocking. If $p, q \in M$ are consecutive conjugate points along a geodesic $\gamma$, then $d(p, q)=0$ or $d(p, q)=\operatorname{Diam}(M, g)$.

Proof Suppose $p, q \in M$ satisfy $0<d(p, q)<\operatorname{Diam}(M, g)$ and let $\gamma:[0,1] \rightarrow M$ be a geodesic with $\gamma(0)=p, \gamma(1)=q$, and along which $q$ is the first conjugate point 
to $p$. Then $\gamma(t)=\exp _{p}(t v)$ for some $v \in T_{p}(M)$. By continuity of the exponential map and the cross blocking property, there is an open set $U \subset T_{p}(M)$ containing $v$ and satisfying $U \subset T^{\prime} \cap T_{p}(M)$. By Proposition 2.3, the points $p$ and $q$ are not consecutive conjugate points, a contradiction.

\section{Blocking light and round spheres}

In this section, we show under various hypothesis that compact Riemannian manifolds with blocking properties similar to those of round spheres are necessarily isometric to round spheres. In general, we believe the following should be true:

Conjecture Let $(M, g)$ be a compact simply connected Riemannian manifold with cross blocking. Then $(M, g)$ is isometric to a simply connected compact rank one symmetric space.

We begin by reviewing the definition and basic properties concerning cut points in a compact Riemannian manifold $(M, g)$. In this section, a unit speed geodesic $\gamma:\left[0, L_{\gamma}\right] \rightarrow M$ for which $\gamma(0)=\gamma\left(L_{\gamma}\right)=p$ will be called a geodesic lasso based at $p$. For a geodesic lasso $\gamma$, we shall denote by $\gamma^{-1}:\left[0, L_{\gamma}\right] \rightarrow M$ the geodesic lasso obtained by traversing $\gamma$ in the reverse direction; specifically, $\gamma^{-1}(t):=\gamma\left(L_{\gamma}-t\right)$. If in addition the geodesic $\gamma$ is regular at $p$, ie, $\dot{\gamma}(0)=\dot{\gamma}\left(L_{\gamma}\right), \gamma$ will be called a closed geodesic based at $p$. By a simple lasso (resp. simple closed geodesic) based at $p$ we mean a lasso (resp. closed geodesic) $\gamma:\left[0, L_{\gamma}\right] \rightarrow M$ based at $p$ which is injective on the interval $\left(0, L_{\gamma}\right)$ and with $p \notin \gamma\left(\left(0, L_{\gamma}\right)\right)$.

Definition Let $(M, g)$ be a compact Riemannian manifold, $p \in M, v \in U_{p}(M)$, and $\gamma:[0, \infty) \rightarrow M$ the unit speed geodesic ray defined by $\gamma(t)=\exp _{p}(t v)$. Let $\left[0, t_{0}\right]$ be the largest interval for which $t \in\left[0, t_{0}\right]$ implies $d(p, \gamma(t))=t$. The point $\gamma\left(t_{0}\right)$ is said to be a cut point to $p$ along the geodesic $\gamma$. The union of the cut points to $p$ along all the geodesics starting from $p$ is called the cut locus and will be denoted by $\operatorname{Cut}(p)$.

The next two propositions are well known and describe points in the cut locus (see eg, do Carmo [6]).

Proposition 3.1 Suppose that $\gamma\left(t_{0}\right)$ is the cut point of $p=\gamma(0)$ along a geodesic $\gamma$. Then either:

- $\gamma\left(t_{0}\right)$ is the first conjugate point of $\gamma(0)$ along $\gamma$, or 
- there exists a geodesic $\sigma \neq \gamma$ from $p$ to $\gamma\left(t_{0}\right)$ such that length $(\sigma)=\operatorname{length}(\gamma)$.

Conversely, if either of these two conditions is satisfied, then there exists $t^{\prime} \in\left(0, t_{0}\right]$ such that $\gamma\left(t^{\prime}\right)$ is the cut point of $p$ along $\gamma$.

Proposition 3.2 Let $p \in M$ and suppose that $q \in \operatorname{Cut}(p)$ satisfies $d(p, q)=$ $d(p, \operatorname{Cut}(p))$. Then either:

- there exists a minimizing geodesic $\gamma$ from $p$ to $q$ along which $q$ is conjugate to $p$, or

- there exist exactly two minimizing geodesics $\gamma$ and $\sigma$ from $p$ to $q$ that together form a simple geodesic lasso based at $p$ of length $2 d(p, \operatorname{Cut}(p))$.

It follows from Proposition 3.1 that $\exp _{p}$ is injective on a ball of radius $r$ centered at the origin in $T_{p}(M)$ if and only if $r<d(p, \operatorname{Cut}(p))$.

Definition The injectivity radius of $(M, g)$ is defined to be

$$
\operatorname{inj}(M, g)=\inf _{p \in M} d(p, \operatorname{Cut}(p))
$$

Note that the injectivity radius of a compact Riemannian manifold is never larger than its diameter. Compact manifolds for which the injectivity radius equals the diameter are known as Blaschke manifolds. All of the compact rank one symmetric spaces are Blaschke and the well known Blaschke conjecture asserts that these are the only Blaschke manifolds of dimension at least two. We will use the following theorem from Berger [2, Appendix D], extending earlier work of Green [8]:

Theorem 3.3 (Berger) Let $(M, g)$ be a Blashke metric on a smooth sphere. Then the metric $g$ is a symmetric metric.

For a Blaschke manifold $(M, g)$, all infinite geodesics $\gamma: \mathbb{R} \rightarrow M$ cover simple closed geodesics of length $2 \operatorname{Diam}(M, g)$ (see [2, Corollary 5.42]). The next proposition is a first step in showing that geodesics in a manifold with cross blocking behave similarly to those in a Blaschke manifold.

Proposition 3.4 Suppose that $(M, g)$ has cross blocking. Let $\gamma:\left[0, L_{\gamma}\right] \rightarrow M$ be a unit speed simple geodesic lasso based at $p \in M$. Then $L_{\gamma} \leq 2 \operatorname{Diam}(M, g)$ and the point $\gamma\left(L_{\gamma} / 2\right)$ is the cut point to $p$ in both of the directions $\dot{\gamma}(0)$ and $-\dot{\gamma}\left(L_{\gamma}\right)$. 
Proof Let $D:=\operatorname{Diam}(M, g)$ and let $c_{1}$ be the cut point to $p$ in the direction $\dot{\gamma}(0)$ and $c_{2}$ be the cut point to $p$ in the direction $-\dot{\gamma}\left(L_{\gamma}\right)$. By simplicity of $\gamma$, there exists unique $t_{1}, t_{2} \in\left(0, L_{\gamma}\right)$ such that $c_{i}=\gamma\left(t_{i}\right)$ for $i=1,2$. By Proposition 3.1, $t_{1} \leq L_{\gamma} / 2 \leq t_{2}$. The statement of the proposition follows from showing that $t_{1}=t_{2}$, ie, that $c_{1}=c_{2}$ and the point $\gamma\left(L_{\gamma} / 2\right)$ is the cut point to $p$ in both of the directions $\dot{\gamma}(0)$ and $-\dot{\gamma}\left(L_{\gamma}\right)$.

We now will assume that $t_{1}<t_{2}$ and must obtain a contradiction. With this assumption, we first argue that $d\left(p, \gamma\left(\left(t_{1}, t_{2}\right)\right)\right)=D$. If not, choose a $t \in\left(t_{1}, t_{2}\right)$ for which $0<d(p, \gamma(t))<D$. Note that since $\gamma$ is simple, the restrictions of $\gamma$ to the interval $[0, t]$ and $\gamma^{-1}$ to the interval $\left[0, L_{\gamma}-t\right]$ define distinct elements in $L_{g}(p, \gamma(t))$ with nonintersecting interiors. There must be a single blocking point on the restriction of $\gamma$ to the interior of each of these intervals since $(M, g)$ has cross blocking. As neither of these light rays are minimizing, there is a unit speed minimizing geodesic $\sigma:\left[0, L_{\sigma}\right] \rightarrow M$ joining $p$ to $\gamma(t)$. Since $\sigma$ is minimizing, it defines a third light ray between $p$ and $\gamma(t)$, whence its interior must intersect one of the two blocking points for $L_{g}(p, \gamma(t))$. Let $s^{\prime}:=\inf \left\{s \in\left(0, L_{\sigma}\right) \mid \sigma(s) \in \operatorname{int}(\gamma)\right\}$. By simplicity of $\gamma$, there is a unique $t^{\prime} \in\left(0, L_{\gamma}\right)$ such that $\sigma\left(s^{\prime}\right)=\gamma\left(t^{\prime}\right):=q$. Since $\sigma$ is unit speed and minimizing, $0<d(p, q)=s^{\prime}<L_{\sigma}=d(p, \gamma(t))<D$, so that $b_{g}(p, q) \leq 2$ by the cross blocking condition. However, the restriction of $\gamma$ to $\left[0, t^{\prime}\right], \gamma^{-1}$ to $\left[0, L_{\gamma}-t^{\prime}\right]$, and the restriction of $\sigma$ to $\left[0, s^{\prime}\right]$ define three distinct elements in $L_{g}(p, q)$ with nonintersecting interiors, implying $b_{g}(p, q) \geq 3$. This is a contradiction, whence $d\left(p, \gamma\left(\left(t_{1}, t_{2}\right)\right)\right)=D$.

Next we show that $d\left(p, \gamma\left(\left(t_{1}, t_{2}\right)\right)\right)=D$ yields a contradiction, completing the proof. By the discreteness of conjugate points along geodesics, there is a $t \in\left(t_{1}, t_{2}\right)$ so that $q:=\gamma(t)$ is not conjugate to $p$ in the direction $\dot{\gamma}(0)$ or in the direction $-\dot{\gamma}\left(L_{\gamma}\right)$. Neither of the restrictions of $\gamma$ to $[0, t]$ or $\gamma^{-1}$ to $\left[0, L_{\gamma}-t\right]$ is minimizing so that there is a unit speed minimizing geodesic $\sigma:[0, D] \rightarrow M$ joining $p$ to $q$. Note that since $\sigma$ is minimizing, the interior of $\sigma$ cannot intersect $\gamma$. Indeed, a first point of intersection between the interiors of $\sigma$ and $\gamma$ would be at a point $p^{\prime}$ satisfying $0<d\left(p, p^{\prime}\right)<D$ so that the reasoning from the previous paragraph may be applied to obtain a contradiction. Let $q_{n}=\sigma\left(D-1 / 2^{n}\right)$. Choose sufficiently small neighborhoods $B_{1}$ of $t \dot{\gamma}(0)$ and $B_{2}$ of $-\left(L_{\gamma}-t\right) \dot{\gamma}\left(L_{\gamma}\right)$ on which $\exp _{p}$ restricts to a local diffeomorphism. For all sufficiently large $n$, there are unique $x_{n} \in B_{1}$ and $y_{n} \in B_{2}$ such that $q_{n}=\exp _{p}\left(x_{n}\right)=\exp _{p}\left(y_{n}\right)$. It follows by the continuity properties of the exponential map that for suitably large $n$, the geodesics

$$
s \mapsto \exp _{p}\left(s x_{n}\right) \quad \text { and } \quad s \mapsto \exp _{p}\left(s y_{n}\right)
$$

for $s \in[0,1]$ and the restriction of $\sigma$ to the interval $\left[0, D-1 / 2^{n}\right]$ define three light rays between $p$ and $q_{n}$ with nonintersecting interior. Hence, $b_{g}\left(p, q_{n}\right) \geq 3$ for suitably large $n$. But $0<d\left(p, q_{n}\right)<D$, so that $b_{g}\left(p, q_{n}\right) \leq 2$ by cross blocking, a contradiction. 
Lemma 3.5 Suppose that $(M, g)$ has cross blocking and sphere blocking. Suppose that $\gamma:\left[0, L_{\gamma}\right] \rightarrow M$ is a unit speed simple geodesic lasso based at $p \in M$. If $L_{\gamma}<2 \operatorname{Diam}(M, g)$, then $\gamma$ is regular at $p$ and all lassos based at $p$ finitely cover $\gamma$. If $L_{\gamma}=2 \operatorname{Diam}(M, g)$, then the interior of all of the geodesic lassos through $p$ intersect in the point $\gamma\left(L_{\gamma} / 2\right)$.

Proof Let $\bar{p}:=\gamma\left(L_{\gamma} / 2\right)$. Suppose there is a (not necessarily simple) unit speed lasso $\tau:\left[0, L_{\tau}\right] \rightarrow M$ through $p$ with $\dot{\tau}(0)$ distinct from $\dot{\gamma}(0)$ and $-\dot{\gamma}\left(L_{\gamma}\right)$. As $(M, g)$ has sphere blocking and $\gamma$ is simple, there is a unique $t \in\left(0, L_{\gamma}\right)$ such that $\gamma(t)$ blocks $L_{g}(p, p)$. Let $s:=\inf \left\{t \in\left(0, L_{\tau}\right] \mid \tau(t)=p\right\}$. The restriction of $\tau$ to the interval $[0, s]$ gives an element in $L_{g}(p, p)$ so that by sphere blocking, its interior must pass through the blocking point $\gamma(t)$ (and hence $\operatorname{int}(\gamma)$ ). Let $s^{\prime}:=\inf \{t \in(0, s) \mid \tau(t) \in \operatorname{int}(\gamma)\}$. By simplicity of $\gamma$ there is a unique $t^{\prime} \in\left(0, L_{\gamma}\right)$ such that $\gamma\left(t^{\prime}\right)=\tau\left(s^{\prime}\right):=q$. As $\gamma$ is simple, the restrictions of $\gamma$ to the intervals $\left[0, t^{\prime}\right], \gamma^{-1}$ to the interval $\left[t^{\prime}, L_{\gamma}\right]$, and $\tau$ to the interval $\left[0, s^{\prime}\right]$ define three distinct light rays between $p$ and $q$ with nonintersecting interiors. Since $p \neq q$ cross blocking implies that $d(p, q)=\operatorname{Diam}(M, g)$ and that $q=$ $\bar{p}$ (by Proposition 3.4 and since $L_{\gamma} / 2 \leq \operatorname{Diam}(M, g)$ ). Hence, if $L_{\gamma} / 2<\operatorname{Diam}(M, g)$ there are no geodesic lassos through $p$ with initial tangent vector outside of the set $\left\{\dot{\gamma}(0),-\dot{\gamma}\left(L_{\gamma}\right)\right\}$, and if $L_{\gamma} / 2=\operatorname{Diam}(M, g)$, the interior of any lasso through the point $p$ passes through the point $\bar{p}$. This concludes the proof of the last statement in the lemma.

We now assume that $L_{\gamma} / 2<\operatorname{Diam}(M, g)$, and will argue that $\gamma$ is regular at the point $p$ and that all lassos at $p$ finitely cover $\gamma$. By simplicity of $\gamma$, the restriction of $\gamma$ to the intervals $\left[0, L_{\gamma} / 2\right]$ and $\gamma^{-1}$ to the interval $\left[0, L_{\gamma} / 2\right]$ define distinct light rays between $p$ and $\bar{p}$ with nonintersecting interiors. As $(M, g)$ has cross blocking, the interior of a third light ray must intersect the interior of $\gamma$ in a blocker and will therefore have a first point of intersection $p^{\prime}$ with the interior of $\gamma$. This implies $b_{g}\left(p, p^{\prime}\right) \geq 3$, a contradiciton. Therefore $\left|L_{g}(p, \bar{p})\right|=2$, while $G_{g}(p, \bar{p})$ is infinite by [24]. Note that any geodesic segment from $G_{g}(p, \bar{p})-L_{g}(p, \bar{p})$ is obtained from extending one of the two light rays in $L_{g}(p, \bar{p})$. Each such extension gives rise to a geodesic lasso based at $p$ with initial tangent vector in the set $\left\{-\dot{\gamma}(0), \dot{\gamma}\left(L_{\gamma}\right)\right\}$. But by the previous paragraph, the initial tangent vector of all lassos at $p$ lie in the set $\left\{\dot{\gamma}(0),-\dot{\gamma}\left(L_{\gamma}\right)\right\}$. Therefore, $\gamma$ must be regular at $p$ and all lassos at $p$ finitely cover $\gamma$.

Definition A $S C_{2 L}$ manifold is a Riemannian manifold with the property that all geodesics cover simple closed geodesics of length $2 L$.

It is tempting to think that the only $S C_{2 L}$ manifolds are the CROSSes. Amazingly, O Zoll exhibited an exotic $S C_{2 L}$ real analytic Riemannian metric on the two sphere 
[27]. This example is discussed in [2, Chapter 4] along with examples on higher dimensional spheres. We remark that these examples are not cross blocked. Indeed, Proposition 3.4 implies that $S C_{2 L}$ manifolds with cross blocking are Blaschke with $\operatorname{inj}(M, g)=\operatorname{Diam}(M, g)=L$, while Theorem 3.3 asserts that there are no exotic Blaschke metrics on spheres. In view of our conjecture that the manifolds with cross blocking are precisely the CROSSes and the Blaschke conjecture that the Blaschke manifolds are precisely the CROSSes, we expect that Blaschke manifolds are precisely those manifolds with cross blocking. In the next proposition, we use well known results concerning Blaschke manifolds to show that Blaschke manifolds all have cross blocking.

Proposition 3.6 Suppose that $(M, g)$ is a Blaschke manifold. Then $(M, g)$ has cross blocking.

Proof Suppose that $p, q \in M$ satisfy $0<d(p, q)<D:=\operatorname{Diam}(M, g)$. By $[2$, Corollary 5.42], $(M, g)$ is a $S C_{2 D}$ manifold. It follows that there is a unit speed simple closed geodesic $\gamma:[0,2 D] \rightarrow M$ with $\gamma(0)=p$ and $\gamma(d(p, q))=q$. The restriction of $\gamma$ to the intervals $[0, d(p, q)]$ and $\gamma^{-1}$ to $[0,2 D-d(p, q)]$ give two distinct elements in $L_{g}(p, q)$ with nonintersecting interiors. Hence $b_{g}(p, q) \geq 2$. If $b_{g}(p, q)>2$ then there must be a third unit speed light ray $\beta:\left[0, L_{\beta}\right] \rightarrow M$ joining $p$ to $q$. Note that since $\beta$ is a light ray and since all geodesics are periodic with period $2 D, L_{\beta}<2 D$. Moreover, since $d(p, q)<D=\operatorname{inj}(M, g)$, the restriction of $\gamma$ to the interval $[0, d(p, q)]$ is the unique minimizing geodesic from $p$ to $q$ so that $L_{\beta}>\operatorname{inj}(M, g)=D$. Extend $\beta$ to the simple closed geodesic $\bar{\beta}:[0,2 D] \rightarrow M$. Then the restriction of $\bar{\beta}$ to the interval $\left[L_{\beta}, 2 D\right]$ gives a geodesic joining $q$ to $p$ of length $2 D-L_{\beta}<D=\operatorname{inj}(M, g)$. Hence, there are two minimizing geodesics joining $p$ and $q$, a contradiction. Therefore, $b_{g}(p, q)=2$ and Blaschke manifolds have cross blocking.

Corollary 3.7 Suppose that $(M, g)$ is Blaschke manifold with sphere blocking. Then $(M, g)$ is isometric to a round sphere.

Proof By the last proposition $(M, g)$ has cross blocking. By Lemma 3.5, the interior of all of the simple closed geodesics through $p$ intersect in a single point $p^{\prime}$ satisfying $d\left(p, p^{\prime}\right)=\operatorname{Diam}(M, g)$. By Proposition $3.4, p^{\prime}$ is the cut point to $p$ along all these geodesics. Hence, $\operatorname{dim}(\operatorname{Cut}(p))=0$, from which it follows (see eg [2, Proposition 5.57]) that $M$ is diffeomorphic to a sphere. By Theorem 3.3, $(M, g)$ is a round sphere.

For the proof of the next theorem, we will need the following two definitions: 
Definition For $p \in M$ and $p^{\prime} \in \operatorname{Cut}(p)$, define the link $\Lambda\left(p, p^{\prime}\right) \subset U_{p^{\prime}} M$ by $\Lambda\left(p, p^{\prime}\right)=\left\{-\dot{\gamma}\left(d\left(p, p^{\prime}\right)\right) \mid \gamma\right.$ is a unit speed and minimizing geodesic from $p$ to $\left.p^{\prime}\right\}$.

Definition For $p \in M$ and $U \subset U_{p} M, U$ is said to be a great sphere if $U$ is the intersection of a linear subspace of $T_{p} M$ with $U_{p} M$.

Theorem 3.8 Suppose that $(M, g)$ is a compact Riemannian manifold with regular cross blocking, sphere blocking, and which does not admit a nonvanishing line field. Then $(M, g)$ is isometric to an even dimensional round sphere.

Proof First we argue that $(M, g)$ is a Blaschke manifold.

To obtain a contradiction, suppose that $\operatorname{inj}(M, g)<\operatorname{Diam}(M, g):=D$. We begin by showing that for each point $x \in M$ satisfying $d(x, \operatorname{Cut}(x))<D$ there is a unique simple closed geodesic based at $x$ and this geodesic has length $2 d(x, \operatorname{Cut}(x))$. Indeed, let $x$ satisfy $d(x, \operatorname{Cut}(x))<D$ and $x^{\prime} \in \operatorname{Cut}(x)$ satisfy $d\left(x, x^{\prime}\right)=d(x, \operatorname{Cut}(x))$. By Corollary 2.5 the points $x$ and $x^{\prime}$ are not conjugate so that by Proposition 3.2 there is a simple geodesic lasso $C$ through $x$ of length $2 \operatorname{inj}(M, g)$. By Lemma 3.5, $C$ is a simple closed geodesic through $x$ and is the unique lasso through $x$, as required.

Let $L:=\sup \{d(p, \operatorname{Cut}(p)) \mid p \in M\} \leq D$. If $L<D$, the preceeding paragraph shows that there is a unique closed geodesic $C_{p}$ of length less than $2 D$ through each point $p \in M$. Since all of the $C_{q}$ have lengths uniformly bounded above, whenever a sequence of points $\left\{p_{i}\right\}$ converge to a point $p_{\infty} \in M$, the sequence of closed geodesics $C_{p_{i}}$ converge to a closed geodesic $C_{\infty}$. By the uniqueness of these geodesics, $C_{\infty}=C_{p_{\infty}}$. Therefore, the tangent spaces to these geodesics define a nonvanishing line field on $M$, a contradiction.

Hence, there is a point $p \in M$ satisfying $d(p, \operatorname{Cut}(p))=D$. Such a point is said to have spherical cut locus at $p$ [2, Definition 5.22]. By [2, Proposition 5.44], the link $\Lambda\left(p, p^{\prime}\right) \subset U_{p^{\prime}}(M)$ is a great sphere for each $p^{\prime} \in \operatorname{Cut}(p)$, whence all geodesics through $p$ are periodic of period $2 D$. Now consider a geodesic connecting $p$ to a point $q$ satisfying $d(q, \operatorname{Cut}(q))<D$. This geodesic gives rise to a closed geodesic of length $2 D$ through $q$, while the first paragraph shows that there is a closed geodesic through $p$ of length $2 d(q, \operatorname{Cut}(q))$. This contradicts Lemma 3.5, implying that at every point $q \in M$, we have $d(q, \operatorname{Cut}(q))=D$, and hence concluding the proof that $(M, g)$ is Blaschke.

By Corollary 3.7, $(M, g)$ is isometric to a round sphere. As $M$ does not admit a nonvanishing line field, $(M, g)$ is isometric to an even dimensional round sphere. 
Next, we adapt Klingenberg's estimate on the injectivity radius to obtain the following (see [6, Chapter 13, Proposition 3.4]):

Theorem 3.9 Suppose that $\left(M^{2 n}, g\right)$ is an even dimensional, orientable, Riemannian manifold with positive sectional curvatures. If $(M, g)$ has regular cross blocking, then $(M, g)$ is Blaschke. In particular, if $M$ is diffeomorphic to a sphere or $(M, g)$ has sphere blocking, then $g$ is a round metric on a sphere.

Proof Suppose to the contrary that $\operatorname{inj}(M, g)<\operatorname{Diam}(M, g)$ and choose $p, q \in M$ so that $q \in \operatorname{Cut}(p)$ and $d(p, q)=\operatorname{inj}(M, g)$. By Corollary 2.5, $p$ and $q$ are not first conjugate points along any geodesic. By Propositions 3.1 and 3.2, there is a unit speed simple closed geodesic $C:[0,2 \operatorname{inj}(M, g)] \rightarrow M$ of length $2 \operatorname{inj}(M, g)$ passing through $p=C(0)$ and $q=C(\operatorname{inj}(M, g))$. Since $M$ is orientable and even dimensional, parallel transport along $C$ leaves invariant a vector $v$ orthogonal to $C$ at $C(0)$. The field $v(t)$ along $C(t)$ is the variational field of closed curves $C_{s}(t)$ for $s \in[0, \epsilon)$. As the sectional curvatures are strictly positive, the second variational formula implies that length $\left(C_{s}\right)<\operatorname{length}(C)$ for all small $s>0$. For each $s>0$, let $q_{s}$ be a point of $C_{s}$ at maximum distance from $C_{s}(0)$. Necessarily, $\lim _{s \rightarrow 0} q_{s}=q$ and $d\left(q_{s}, C_{s}(0)\right)<\operatorname{inj}(M, g)$. For each $s>0$, let $\gamma_{s}$ be the unique minimizing geodesic joining $q_{s}$ to $C_{s}(0)$. Note that each $\dot{\gamma}_{s}(0)$ is orthogonal to $C_{s}$ by the first variational formula. Let $w \in T_{q} M$ be an accumulation point of the vectors $\dot{\gamma}_{s}(0) \in T_{q_{s}} M$ and $\gamma:[0,1] \rightarrow M$ be the geodesic defined by $\gamma(t):=\exp _{q}(t w)$. It follows that $\gamma$ is a minimizing geodesic joining $q$ to $p$ which is orthogonal to $C$ at $q$. As $\gamma$ is minimizing, it cannot intersect $C$ except at the points $p$ and $q$, whence $b_{g}(p, q) \geq 3$, a contradiction. Therefore $(M, g)$ is Blaschke. The last statement follows from Theorem 3.3 and Corollary 3.7.

Theorem 3.10 Suppose that $\left(S^{2}, g\right)$ is a Riemannian metric on the two sphere with cross blocking and sphere blocking. Then a shortest nontrivial closed geodesic is simple and has length $2 \operatorname{Diam}\left(S^{2}, g\right)$.

Proof Let $D:=\operatorname{Diam}(M, g)$ and let $C$ be a shortest nontrivial closed geodesic. We first argue that if $C$ is simple, then its length is $2 \operatorname{Diam}\left(S^{2}, g\right)$. Indeed, by Proposition 3.4 , length $(C) \leq 2 D$. We suppose that length $(C)<2 D$ and will obtain a contradiciton. Note that $C$ separates $S^{2}$ into two components. By Santalo's formula (see [23, page 488] or [1, page 290]), almost all geodesic rays with initial point on $C$ eventually leave the component they initially enter. Choose one such ray $\gamma:[0, \infty) \rightarrow M$ and let $t:=\inf \{t \in(0, \infty) \mid \gamma(t) \in C\}$. If $\gamma(t)$ is distinct from $\gamma(0)$, then $b_{g}(\gamma(0), \gamma(t)) \geq 3$, a contradiction. Hence, $\gamma(0)=\gamma(t)$, also a contradiction by Lemma 3.5. 
Next we argue that a shortest nontrivial closed geodesic $C$ is simple. By Nabutovsky and Rotman [20] or Sabourau [22], length $(C) \leq 4 D$. Suppose that $C$ is not simple, and choose a unit speed paramaterization $C:\left[0, L_{C}\right] \rightarrow M$ such that $C(0)$ is a crossing point. Let $s=\inf \left\{t \in\left(0, L_{C}\right) \mid C(s)=C(0)\right\}$. Without loss of generality, we may assume that the restriction of $C$ to the interval $[0, s]$ defines a simple lasso at $C(0)$, whence $s=2 D$ by Proposition 3.4. The restriction of $C$ to the interval $\left[2 D, L_{C}\right]$ defines another lasso at $C(0)$. If this lasso is not simple, then it contains a simple lasso of length $2 D$ and $L_{C}>4 D$, a contradiction. Hence, the restriction of $C$ to the interval $\left[2 D, L_{C}\right]$ defines a simple lasso and $L_{C}=4 D$. By Lemma 3.5, $C(D)=C(3 D)$. Note that the restriction of $C$ to $[0,2 D]$ separates $S^{2}$ into two components. This implies that $C((0,2 D)) \cap C((3 D, 4 D)) \neq \varnothing$. Letting $s=\inf \{t \in(3 D, 4 D) \mid C(t) \in C((0,2 D))\}$, it follows that $b_{g}(C(D), C(s)) \geq 3$, a contradiction.

\section{Finite blocking property and entropy}

In this section we relate the finite blocking property for a compact Riemannian manifold $(M, g)$ to the topological entropy $h_{\text {top }}(g)$ of its geodesic flow.

Our starting point is a well known theorem (see eg, Mañé [15, Corollary 1.2]) identifying the topological entropy with the exponential growth rate of the number of geodesics between pairs of points in $M$. For $x, y \in M$ and $T>0$, let $n_{T}(x, y)\left(\right.$ resp. $\left.m_{T}(x, y)\right)$ denote the number of geodesic segments (resp. light rays) between the points $x$ and $y$ of length no more than $T$.

Theorem 4.1 (Mañé) Let $(M, g)$ be a compact Riemannian manifold without conjugate points. Then

$$
h_{\mathrm{top}}(g)=\lim _{T \rightarrow \infty} \frac{\log \left(n_{T}(x, y)\right)}{T},
$$

for all $(x, y) \in M \times M$.

The main observation of this section lies in the following:

Proposition 4.2 Let $(M, g)$ be a compact Riemannian manifold without conjugate points. If $h_{\text {top }}(g)>0$, then $b_{g}(x, y)=\infty$ for all $(x, y) \in M \times M$.

Proof Let $I:=\operatorname{inj}(M, g)$. We first argue that $n_{T}(x, y) \leq(T / 2 I)^{2} m_{T}(x, y)$. For a unit speed geodesic $\gamma:\left[0, L_{\gamma}\right] \rightarrow M$ in $G_{g}(x, y)$, let $t_{1}(\gamma):=\sup \left\{t \in\left[0, L_{\gamma}\right) \mid \gamma(t)=\right.$ $x\}$ and $t_{2}(\gamma):=\inf \left\{t \in\left(t_{1}(\gamma), L_{\gamma}\right] \mid \gamma(t)=y\right\}$. Restrict $\gamma$ to the interval $\left[t_{1}(\gamma), t_{2}(\gamma)\right]$ and change the parameter of this interval to define a unit speed geodesic

$$
\operatorname{Light}(\gamma):\left[0, t_{2}(\gamma)-t_{1}(\gamma)\right] \rightarrow M
$$


in $L_{g}(x, y)$. Since for each $\beta \in L_{g}(x, y), \operatorname{Light}(\beta)=\beta$, it follows that

$$
\text { Light: } G_{g}(x, y) \rightarrow L_{g}(x, y)
$$

defines a surjective map. To conclude this step, it suffices to show that given a fixed unit speed $\beta \in L_{g}(x, y)$ of length not more than $T>0$, there are at most $(T / 2 I)^{2}$ distinct preimages of $\beta$ under the map Light of length not more than $T$. For each unit speed geodesic $\gamma:\left[0, L_{\gamma}\right] \rightarrow M$ of length not more than $T$ satisfying $\operatorname{Light}(\gamma)=\beta$, $\dot{\gamma}\left(t_{1}(\gamma)\right)=\dot{\beta}(0)$ and $\dot{\gamma}\left(t_{2}(\gamma)\right)=\dot{\beta}\left(L_{\beta}\right)$ since geodesics are determined by their initial conditions. It follows that the number of preimages of $\beta$ having length bounded above by $T$ coincides with the number of different extensions of $\beta$ to a unit speed geodesic $\bar{\beta} \in G_{g}(x, y)$ having length not more that $T$. Given such an extension $\bar{\beta}$, let $n_{\bar{\beta}}(x)$ and $n_{\bar{\beta}}(y)$ be the number of returns to $x$ and the number or returns to $y$. Necessarily, $n_{\bar{\beta}}(x), n_{\bar{\beta}}(y) \leq T / 2 I$ since each return to $x$ or return to $y$ increases the length of $\beta$ by at least $2 I$. Hence, by uniqueness of geodesics, $n_{T}(x, y) \leq(T / 2 I)^{2} m_{T}(x, y)$.

To complete the proof, we argue by contradiction, assuming there is a pair of points $x, y \in M$ with a finite blocking set $F=\left\{b_{1}, \ldots b_{k}\right\} \subset M-\{x, y\}$ for $L_{g}(x, y)$. By definition, the interior of any light ray $\gamma \in L_{g}(x, y)$ passes through some point $b_{i} \in F$, breaking $\gamma$ into two geodesic segments $\gamma_{1} \in G_{g}\left(x, b_{i}\right)$ and $\gamma_{2} \in G_{g}\left(b_{i}, y\right)$. If $\gamma$ has length bounded above by $T$, then one of $\gamma_{1}$ or $\gamma_{2}$ must have length bounded above by $T / 2$. Moreover, given a geodesic segment $\alpha \in G_{g}\left(x, b_{i}\right)$ (resp. $\beta \in G_{g}\left(y, b_{i}\right)$ ), there is at most one extension of $\alpha$ (resp. $\beta$ ) to a light ray between $x$ and $y$. It follows that $m_{T}(x, y) \leq \sum_{j=1}^{k} n_{T / 2}\left(x, b_{j}\right)+n_{T / 2}\left(b_{j}, y\right)$. Combining this with the estimate from the previous paragraph yields:

$$
n_{T}(x, y) \leq(T / 2 I)^{2} \sum_{j=1}^{k} n_{T / 2}\left(x, b_{j}\right)+n_{T / 2}\left(b_{j}, y\right) .
$$

Let $0<\epsilon<h_{\text {top }}(g) / 3$. By Theorem 4.1 , there is a $T_{0} \in \mathbb{R}$ so that $T>T_{o}$ implies

$$
\left|h_{\text {top }}(g)-\frac{\log \left(n_{T}\left(*_{1}, *_{2}\right)\right)}{T}\right|<\epsilon,
$$

for all $*_{1}, *_{2} \in\{x, y\} \cup F$. Therefore

$$
\exp ^{\left(h_{\mathrm{top}}(g)-\epsilon\right) T}<n_{T}\left(*_{1}, *_{2}\right)<\exp ^{\left(h_{\mathrm{top}}(g)+\epsilon\right) T},
$$

for all $T>T_{0}$ and $*_{1}, *_{2} \in\{x, y\} \cup F$. It now follows that

$$
\exp ^{\left(h_{\mathrm{top}}(g)-\epsilon\right) T}<n_{T}(x, y)<2 k(T / 2 I)^{2} \exp ^{\left(h_{\mathrm{top}}(g)+\epsilon\right) T / 2},
$$

a contradiction for all sufficiently large values of $T$. 
We remark that the conclusion $b_{g}(x, y)=\infty$ for all $(x, y) \in M \times M$ may be phrased more geometrically as saying that given any point $(x, y) \in M \times M$ and any finite set $F \subset M-\{x, y\}$, there is a geodesic segment between $x$ and $y$ avoiding $F$.

As a corollary of Proposition 4.2, we obtain the following:

Theorem 4.3 Let $(M, g)$ be a compact Riemannian manifold with nonpositive sectional curvatures. Then $(M, g)$ has finite blocking if and only if $(M, g)$ is flat.

Proof Assume that $(M, g)$ has nonpositive curvature and is not flat. Then $(M, g)$ has no conjugate or focal points. By Pesin [21, Corollary 3], a geodesic flow on a nonflat compact Riemannian manifold without focal points has positive entropy. By Proposition 4.2, $(M, g)$ does not have finite blocking.

K Burns and E Gutkin [4] made the nice observation that by assuming uniform finite blocking and by iterating the line of reasoning used in the proof of Proposition 4.2 one can establish the following:

Theorem 4.4 (Burns-Gutkin) Let $(M, g)$ be a compact Riemannian manifold with the uniform finite blocking. Then $h_{\text {top }}(g)=0$ and $\pi_{1}(M)$ has polynomial growth.

Using their result we obtain the following:

Theorem 4.5 Let $(M, g)$ be a compact Riemannian manifold with regular finite blocking. Then $(M, g)$ is flat.

Proof By Corollary 2.4, $(M, g)$ has uniform finite blocking and is conjugate point free. By Theorem 4.4, $\pi_{1}(M)$ has polynomial growth. By Lebedeva [14], compact Riemannian manifolds without conjugate points and with polynomial growth fundamental group are flat.

\section{Finite blocking property and buildings}

In this section, we provide a proof of Theorem 1, which states that compact quotients of Euclidean buildings have uniform finite blocking. Let us start by recalling some elementary facts about Euclidean buildings, referring the reader to Brown [3] for more details.

Let $W \subset \mathbb{R}^{n}$ be a compact polyhedron, with all faces forming angles of the form $\pi / m_{i j}$ for some positive integer $m_{i j}$. Let $\Lambda \subset \operatorname{Isom}\left(\mathbb{R}^{n}\right)$ be the Coxeter group generated by 
reflections in the faces of the polyhedron, and observe that the $\Lambda$-orbit of $W$ generates a tessellation of $\mathbb{R}^{n}$ by isometric copies of $W$. We can label the faces of the copies of $W$ in the tessellation of $\mathbb{R}^{n}$ according to the face of $W$ whose orbit contains them. A Euclidean building is a polyhedral complex $\tilde{X}$, equipped with a CAT(0)-metric, having the property that each top dimensional polyhedron is isometric to $W$ (these will be called chambers). In addition, a certain number of axioms are required to be satisfied. We omit a precise definition of Euclidean buildings, contenting ourselves with mentioning the properties we will need. The reader may refer to [3] for a precise definition, and to Davis [5] for geometric properties of these buildings. The polyhedral complex must also satisfy:

- Each face of the complex $\tilde{X}$ is labelled with one of the faces of the polyhedron $W$.

- Given any pair of points $x, y \in \tilde{X}$, there exists an isometric, polyhedral, label preserving embedding of the tessellated $\mathbb{R}^{n}$ whose image contains $x$ and $y$. The image of such an embedding is called an apartment.

- The group $\operatorname{Isom}(\tilde{X})$ is defined to be the group of label preserving isometries of $\tilde{X}$.

- Given any two apartments $\mathcal{A}_{1}, \mathcal{A}_{2}$ whose intersection is non-empty, there exists an element $\phi \in \operatorname{Isom}(\tilde{X})$ which fixes pointwise $\mathcal{A}_{1} \cap \mathcal{A}_{2}$, and satisfies $\phi\left(\mathcal{A}_{1}\right)=$ $\mathcal{A}_{2}$.

We will say that $X$ is a compact quotient of $\tilde{X}$ provided it is the quotient of $\tilde{X}$ by a cocompact subgroup of $\operatorname{Isom}(\tilde{X})$, acting fixed point freely.

Note that in a Euclidean building, one has uniqueness of geodesics joining pairs of points (from the CAT(0) hypothesis). Furthermore, we can pick an apartment containing both $x$ and $y$, giving a totally geodesic $\mathbb{R}^{n}$ inside $\tilde{X}$ containing $x, y$. Then the geodesic joining $x$ to $y$ coincides with the straight line segment from $x$ to $y$ within the apartment. We will call the point along the geodesic that is equidistant from $x$ and $y$ the midpoint of $x$ and $y$, and denote it by $(x+y) / 2$.

Another important point is that both the building $\tilde{X}$, as well as the compact quotient $X$ come equipped with a canonical folding map to the canonical chamber $W$, given by the labeling. We will use $\rho$ to denote the canonical folding map, and given a point $x \in \tilde{X}$ (or in $\tilde{X} / \Gamma$ ), we define the type of the point $x$ to be the point $\rho(x) \in W$. We now make two observations:

- In a compact quotient $\tilde{X} / \Gamma$, there are only finitely many points of any given type. 
- Given any point $p$ in $\tilde{X} / \Gamma$, every pre-image of $p$ in the universal cover $\tilde{X}$ has exactly the same type as $p$.

The proof of the theorem will make use of the following easy lemma:

Lemma 5.1 Let $\mathcal{A}$ be any apartment, and $x, y \in W$ a pair of points in the model chamber. Define $\mathcal{S}(x), \mathcal{S}(y)$ to be the set of points in $\mathcal{A}$ of type $x, y$ respectively. Then there exist a finite collection of points $b_{1}, \ldots, b_{k} \in W$ having the property that:

$$
\{(\bar{x}+\bar{y}) / 2 \mid \bar{x} \in \mathcal{S}(x), \bar{y} \in \mathcal{S}(y)\} \subset \bigcup_{i=1}^{k} \mathcal{S}\left(b_{i}\right)
$$

Proof We first observe that the Coxeter group $\Lambda$ contains an isomorphic copy of $\mathbb{Z}^{r}$ as a finite index subgroup, where $r=\operatorname{dim}(\mathcal{A})$. In particular, if we denote by $\Lambda^{\prime}$ this finite index subgroup, we note that each of the two sets $\mathcal{S}(x), \mathcal{S}(y)$ are the union of $\left[\Lambda: \Lambda^{\prime}\right]=m$ disjoint copies of $\Lambda^{\prime}$-orbits in $\mathcal{A}$. Now note that given any two $\Lambda^{\prime}$-orbits in $\mathcal{A}$, the collection of midpoints of points in the first orbit with points in the second orbit lie in a finite collection of $\Lambda^{\prime}$-orbits (in fact, at most $2^{r}$ such orbits). This is immediate from the proof of the fact that a flat torus has finite blocking.

This in turn implies that the collection of midpoints of points in the set $\mathcal{S}(x)$ and points in the set $\mathcal{S}(y)$ lie in the union of at most $2^{r} m^{2}$ of the $\Lambda^{\prime}$-orbits in $\mathcal{A}$. Since each $\Lambda^{\prime}$-orbit lies in a corresponding $\Lambda$-orbit, we conclude that the collection of midpoints lie in the union of a finite collection of $\Lambda$-orbits. But two points are in the same $\Lambda$-orbit if and only if they have the same type. Hence choosing the points $b_{1}, \ldots, b_{k}$ to be the finitely many types (at most $2^{r} m^{2}$ of them), we get the desired containment of sets.

We now proceed to prove Theorem 1:

Proof of Theorem 1 Let $X=\tilde{X} / \Gamma$ be a compact quotient of the Euclidean building $\tilde{X}$, and let $W$ denote a model chamber. Given two points $x, y$ in the space $X$, we want to exhibit a finite set of blockers. Consider the sets $\mathcal{P}(x), \mathcal{P}(y) \subset \tilde{X}$ consisting of all pre-images of the points $x, y$, respectively, under the covering map $\tilde{X} \rightarrow X=\tilde{X} / \Gamma$. As we previously remarked, we can make sense of the midpoint of a pair of points in $\tilde{X}$. We now claim that the collection of midpoints joining points in $\mathcal{P}(x)$ to points in $\mathcal{P}(y)$ have only a finite number of possible types in $W$.

In order to see this, let us apply the previous lemma to the types $\rho(x), \rho(y) \in W$. First note that given an arbitrary pair of points $\bar{x} \in \mathcal{P}(x), \bar{y} \in \mathcal{P}(x)$, there exists an apartment $\mathcal{A}$ containing $\bar{x}, \bar{y}$. Furthermore, we have the obvious containments 
$\mathcal{P}(x) \cap \mathcal{A} \subset \mathcal{S}(\rho(x)), \mathcal{P}(y) \cap A \subset \mathcal{S}(\rho(y))$, and hence the midpoint joining $\bar{x}$ to $\bar{y}$ has the property that its type is one of the finitely many points $b_{1}, \ldots, b_{k}$.

Hence to obtain a finite blocking set, let us consider the collection of all points in $X$ whose type is one of $b_{1}, \ldots, b_{k}$. This yields a finite collection $\mathcal{B}$ of points in $X$ having the property that if $\gamma$ is an arbitrary geodesic joining $x$ to $y$, its midpoint must be one of the points in $\mathcal{B}$, completing the proof of the theorem.

\section{References}

[1] M Berger, Lectures on geodesics in Riemannian geometry, Tata Institute of Fundamental Research Lectures on Mathematics 33 (1965) MR0215258

[2] A L Besse, Manifolds all of whose geodesics are closed, Ergebnisse series 93, Springer, Berlin (1978) MR496885 With appendices by D B A Epstein, J-P Bourguignon, L Bérard-Bergery, M Berger and J L Kazdan

[3] KS Brown, Buildings, Springer Monographs in Mathematics, Springer, New York (1998) MR1644630 Reprint of the 1989 original

[4] K Burns, E Gutkin, Growth of the number of geodesics between points and insecurity for Riemannian manifolds arXiv:math.DS/0701579

[5] M W Davis, Buildings are CAT(0), from: "Geometry and cohomology in group theory (Durham, 1994)”, London Math. Soc. Lecture Note Ser. 252, Cambridge Univ. Press (1998) 108-123 MR1709955

[6] MP do Carmo, Riemannian geometry, Mathematics: Theory \& Applications, Birkhäuser, Boston (1992) MR1138207 Translated from the second Portuguese edition by F Flaherty

[7] D Fomin, Zadaqi Leningradskih Matematitcheskih Olimpiad (Leningrad 1990)

[8] L W Green, Auf Wiedersehensfächen, Ann. of Math. (2) 78 (1963) 289-299 MR0155271

[9] E Gutkin, Blocking of billiard orbits and security for polygons and flat surfaces, Geom. Funct. Anal. 15 (2005) 83-105 MR2140629

[10] E Gutkin, Insecure configurations in lattice translation surfaces, with applications to polygonal billiards, Discrete Contin. Dyn. Syst. 16 (2006) 367-382 MR2226486

[11] E Gutkin, Blocking of orbits and the phenomenon of (in)security for the billiard in polygons and flat surfaces, preprint IHES/M/03/06

[12] E Gutkin, V Schroeder, Connecting geodesics and security of configurations in compact locally symmetric spaces, Geom. Dedicata 118 (2006) 185-208 MR2239456

[13] P Hiemer, V Snurnikov, Polygonal billiards with small obstacles, J. Statist. Phys. 90 (1998) 453-466 MR1611100 
[14] N D Lebedeva, Spaces without conjugate points and with fundamental group of polynomial growth, Algebra i Analiz 16 (2004) 69-81 MR2068342 Russian; English translation: St. Petersburg Math. J. 16 (2005) 341-348

[15] R Mañé, On the topological entropy of geodesic flows, J. Differential Geom. 45 (1997) 74-93 MR1443332

[16] T Monteil, A counter-example to the theorem of Hiemer and Snurnikov, J. Statist. Phys. 114 (2004) 1619-1623 MR2039490

[17] T Monteil, Finite blocking versus pure periodicity arXiv:math.DS/0406506

[18] T Monteil, On the finite blocking property, Ann. Inst. Fourier (Grenoble) 55 (2005) 1195-1217 MR2157167

[19] T Monteil, A homological condition for a dynamical and illuminatory classification of torus branched coverings arXiv:math. DS/060335

[20] A Nabutovsky, R Rotman, The length of the shortest closed geodesic on a 2dimensional sphere, Int. Math. Res. Not. (2002) 1211-1222 MR1903953

[21] J B Pesin, Formulas for the entropy of the geodesic flow on a compact Riemannian manifold without conjugate points, Mat. Zametki 24 (1978) 553-570, 591 MR513656 Russian; English translation: Math. Notes 24 (1978) 796-805

[22] S Sabourau, Filling radius and short closed geodesics of the 2-sphere, Bull. Soc. Math. France 132 (2004) 105-136 MR2075918

[23] L A Santaló, Integral geometry in general spaces, from: "Proceedings of the ICM (Cambridge, MA, 1950) Vol. 1”, Amer. Math. Soc. (1952) 483-489 MR0044146

[24] J-P Serre, Homologie singulière des espaces fibrés. Applications, Ann. of Math. (2) 54 (1951) 425-505 MR0045386

[25] F W Warner, The conjugate locus of a Riemannian manifold, Amer. J. Math. 87 (1965) 575-604 MR0208534

[26] F W Warner, Conjugate loci of constant order, Ann. of Math. (2) 86 (1967) 192-212 MR0214005

[27] O Zoll, Ueber Flächen mit Scharen geschlossener geodätischer Linien, Math. Ann. 57 (1903) 108-133 MR1511201

Department of Mathematics, The Ohio State University

Columbus, $\mathrm{OH} 43210$, USA

Department of Mathematics, University of Chicago

5734 S University Avenue, Chicago, IL 60637, USA

jlafont@math.ohio-state.edu, schmidt@math.uchicago.edu

Proposed: Benson Farb

Seconded: Walter Neumann, Tobias Colding
Received: 3 August 2006

Accepted: 21 March 2007 